\title{
Predictive Performance of Gargle Test in Prediction of Successful Extubation in Patients Undergoing Head and Neck Surgery in ICU
}

Mohammad Taghi Beigmohammadi ( $\sim$ mbage46@gmail.com )

TUMS: Tehran University of Medical Sciences https://orcid.org/0000-0002-1925-8760

Laya Amoozadeh

TUMS: Tehran University of Medical Sciences https://orcid.org/0000-0002-1073-3529

Abbas Alipour

Mazandaran University of Medical Sciences

\section{Research}

Keywords: Exubation Failure, Cuff Leak Test, Gargle, Critical Care

Posted Date: May 17th, 2021

DOl: https://doi.org/10.21203/rs.3.rs-490228/v1

License: (c) (i) This work is licensed under a Creative Commons Attribution 4.0 International License.

Read Full License 


\section{Abstract \\ Background}

Improvement of predictive tools for recognition of airway edema is crucial for safe extubation and patient safety. This study aims to evaluate the efficacy of gargle test for assessment of airway edema and prediction of successful extubation in patients undergoing head and neck surgeries.

\section{Methods}

This is a prospective observational study on 118 patients that were undergone head and neck surgeries and been admitted to intensive care units. All the patients were weaned based on the same protocol. Initially, quantitative, and qualitative Cuff Leak Test were done and, the decision for extubation was made by employing them. Subsequently, gargling with purified water was implemented. Exclusion criteria were consisted of the impossibility of extubation due to reasons other than airway edema such as pneumonia. Independent sample t-test was applied to study the difference between quantitative variables between two groups, and Fisher's exact test was used for categorical variables. P-value $<0.05$ was determined as a significant difference between the variables. To investigate the relationship between these two tests, sensitivity, specificity, positive and negative predictive values, and accuracy were calculated.

\section{Result}

One hundred eighteen were enrolled in this study. Sixty-seven patients $(56.78 \%)$ male and 51 patients $(43.22 \%)$ were female with a mean age of $54.7 \pm 12.2$ year. Due to surgical manipulations, and anatomical problems in the hypopharynx and larynx, 5 patients $(4.2 \%)$ were not able to perform the gargle test. Six patients (5.1\%) were facing extubation failure since they experienced respiratory distress and stridor. A measure of agreement (kappa) of gargle test with qualitative and quantitative CLT was low $(\mathrm{K}=0.21, \mathrm{k}=0.07$, respectively). The gargle test had higher specificity, negative predictive value (NPV), and accuracy compared to quantitative and qualitative CLT. Extubation failure in patients with positive gargle test was significantly lower than patients with negative gargle test (3.7\% vs. $33.4 \%$ respectively, P $=0.032$ ).

\section{Conclusion}

The gargle test is simple, cheap, and does not require any equipment, and groups of nerves and muscles which control larynx function are being assessed in the gargle test. It provides better patients safety in difficult extubations.

\section{Background}


The decision to extubate the patients in the intensive care unit (ICU) is critical and challenging. On the one hand, delay in the removal of endotracheal tubes is associated with complications such as infection and increased mortality, on the other hand, inappropriate decision to extubate the patient with anatomical problems, trauma, head and neck surgeries, restricted mouth opening, and neck limited range of motion can result in difficult intubation which eventually can be life-threatening. (1) Thus, improvement of predictive tools and recognition of airway edema is crucial for safe extubation, and it is necessary to predict if the patient can tolerate extubation and if reintubation is still a possibility. $(2,3,4)$. Improvement of these methods is required for patient safety, as it helps the intensivist to have a clearer evaluation and make a more appropriate decision for extubation in difficult situations. $(3,4)$

Studies have reported the prevalence of laryngeal edema; $0.6-36.8 \%$, reintubation; up to $80 \%$ (5), mortality; $30-40 \%$, and increased pulmonary complications in difficult extubation. (6) This statistical discrepancy variabilities could be due to vocal cord dysfunction or laryngeal edema, reduced tracheal cross-sectional area, different study populations, and duration of intubation. $(5,6,7)$

Unlike tongue and pharynx edema, evaluation of laryngeal edema is difficult. Female gender, prolonged intubation, the severity of the airway edema, endotracheal tube size compared to larynx are associated with a higher risk of stridor after extubation and cause false negative or positive results in cuff leak test. $(8,9)$

Considering the impacts of numerous confounding factors, false results, the discrepancy between the severity of clinical presentation of airway edema with the extent of tissue injury, and variable results in various measurements(9), it is essential to develop more reliable methods in airway evaluation. Based on this, physicians are attempting to employ various techniques to overcome this issue. These techniques include cuff leak test (quantitative/qualitative) (9), tracheal ultrasound (10), optic fibroscopy, direct laryngoscopy examination, and video laryngoscopy $(11,12)$, and flow-volume curves for extra-thoracic stenosis. (13)

Although tongue and pharyngeal edema can be easily evaluated via direct laryngoscopy, assessment of laryngeal edema and its severity in the presence of endotracheal tube is challenging. Airway studies with video laryngoscopy and fiberoptic bronchoscopy require patient extubation that can be life-threatening. (8) Cuff leak test is a simple method to predict the occurrence of complications. However, its result can be impacted by confounding factors, such as the ratio of the tube to trachea size, pulmonary mechanics, and rate of airflow. $(7,14)$ Optimally, the test should be used just before extubation for every patient. $(8$, 13) Due to numerous reasons, every single method mentioned above has limitations, as they require equipment, performer skills, and are associated with limited effectiveness or necessitate removal of the tube. Imaging techniques such as plain cervical radiography $(15,16)$, CT scan, and MRI can capture upper airway edema; However, they are expensive and impractical in clinical practice, and they are also accompanied by technical limitations. (13) Gargle is holding water in the back of the mouth and blowing air out from the lungs by utilization of groups of nerves and muscles in upper airways with a gurgling 
sound. Thus, this study is conducted to evaluate the efficacy of the gargle test for assessment of airway edema and prediction of successful extubation in patients undergoing head and neck surgeries.

\section{Methods}

\section{Study population and setting}

After review and approval by the university ethics committee (IR.TUMS.IKHC.REC.1396.2004), a prospective observational study was conducted. One hundred eighteen patients were investigated; they had undergone head and neck surgeries and been admitted in general and surgical intensive care units in Imam Khomeini Hospital Complex of Tehran University of Medical Sciences. All patients were intubated and supported by mechanical ventilation for at least 12 hours.

Exclusion criteria were consisted of the impossibility of extubation due to reasons other than airway edema, pneumonia, or other reasons that prohibited ventilator weaning.

\section{Weaning and airway assessment:}

Airway management and monitor of the mechanical ventilator in the intensive care unit were accomplished under an intensivist's supervision. All the patients had endotracheal tubes with low pressure-high volume cuffs, and cuff pressure was monitored and maintained less than $25 \mathrm{cmH} 20$ every 4 hours. Weaning patients from the ventilator was on the grounds of a consistent protocol utilizing pressure support mode. Once the patient met all the criteria of ventilator weaning, the decision for extubation was made by employing Cuff Leak Test (CLT). Initially, quantitative, and qualitative CLT were done. Subsequently, gargling with purified water was implemented. Patients were given the essential explanations to mentally prepare them, improve their cooperation, and obtain their consent. Whenever it was necessary, an appropriate dose of a sedative was applied to enhance the patient's comfort and tolerance to an endotracheal tube. None of the patients were on corticosteroid drugs.

Quantitative CLT: Following suction of oral and tracheal secretions, the patient was placed in a sitting position. Prior to the test, the patient was put on the assist-control ventilation mode with a tidal volume of 6-8 $\mathrm{ml} / \mathrm{kg}$ for 2 minutes. The average exhaled tidal volume was recorded over the next 5 respiratory cycles before and after cuff deflation. A difference of more than $15 \%$ in the tidal volume was associated with the absence of airway edema and warranted the removal of the tube. (17)

Qualitative CLT: First the patient was weaned from the ventilator. Then, the oral and tracheal secretions were suctioned. Subsequently given that the patient was comfortable and placed in a sitting position, the endotracheal cuff was deflated. Next, the opening of the endotracheal tube was blocked and the patient was asked to breathe through the mouth. If the patient was able to perform 5 respiratory cycles without any sign of suffocation or evidence of airway obstruction, it was deemed as an appropriate test.

Gargle Test (GT): Like the qualitative CLT method, the patient was weaned from the ventilator in the sitting position. After performing oral and tracheal suction, the patient did a relatively deep inspiration 
and held his/her breath. Then, concurrent breath-hold, the endotracheal tube was occluded and about 3$5 \mathrm{ml}$ of purified water was put in the patient mouth. Next, the patient was asked to gargle water at the back of his mouth with long and slow exhalation. This indicates that there is an appropriate air leak (in terms of volume, flow, and power) around the tube. Also, it demonstrates the ability of the patient in maintaining airway patency and prevention from aspiration. Then, the patient was asked to lean forward and spit the water out in a dish.

The extubation decision was based on the result of CLT. All tests were done by another anesthesiologist and he allowed extubation. Extubation was done by the patient's nurse. The investigator did not know the result of the gargle test and just recorded data immediately after extubation such as stridor, dyspnea, and the patient's ability for talking. The need for early reintubation in the next 12 hours due to respiratory distress caused by airway edema and stridor considered extubation failure.

Age, gender, type of the surgery, duration of anesthesia and surgery, comorbidities, duration of mechanical ventilation (from initiation of anesthesia to extubation), type of intubation, success, or failure of extubation, and results of quantitative, qualitative CLT, and gargle test were documented.

\section{Statistical Analysis}

A minimum value of the correlation coefficient between the gargle test and quantitative/qualitative CLT was defined as 0.6. Initially, Spearman's correlation coefficient and later kappa coefficient (with 95\% confidence interval) were calculated to study Inter-rater reliability between gargle test and quantitative/qualitative CLT. To investigate the relationship between these two tests, sensitivity, specificity, positive and negative predictive values, and accuracy were calculated. Data are demonstrated in the form of mean \pm standard deviation. Frequency was used to describe qualitative variables. Independent sample t-test was applied to study the difference between quantitative variables between two groups, and Chi-square or Fisher exact test was used for categorical variables. P-value $<0.05$ was determined as a significant difference between the variables.

A ROC curve was used to identify the cut-off value for the minimum percentage of an acceptable leak for removal of the endotracheal tube in this study.

\section{Results}

One hundred eighteen patients who had undergone head and neck cancer surgeries including thyroidectomy, neck dissection, glossectomy, mandibulectomy, maxillectomy were enrolled in this study. Sixty-seven patients $(56.78 \%)$ male and 51 patients (43.22\%) were female with a mean age of $54.7 \pm$ 12.2. Demographic characteristics of the patients are depicted in (Table 1). Seventy-six patients (64.4\%) were nasally intubated, while 42 patients (35.6\%) were orally intubated. Due to surgical manipulations, and anatomical problems in the hypopharynx and larynx, 5 patients $(4.2 \%)$ were not able to perform the gargle test. These 5 patients were excluded from data analysis and 113 patients were enrolled for analysis. Six patients (5.1\%) were facing extubation failure since they experienced respiratory distress 
and stridor. Extubation failure was not statistically significant based on the result of quantitative and qualitative CLT ( $P=0.647, P=0.385$, respectively) (Table 2). However, extubation failure in patients with positive gargle test was significantly lower than patients with negative gargle test (3.7\% vs. $33.4 \%$ respectively, $\mathrm{P}=0.032$ ) (Table 2). A measure of agreement (kappa) of gargle test with qualitative and quantitative CLT was low ( $K=0.21, k=0.07$, respectively). The gargle test had higher specificity, negative predictive value (NPV), and accuracy compared to quantitative and qualitative CLT (Table 3).

According to ROC curve, cut- off value (Fig. 1: A point) in quantitative CLT was predicted $16.5 \%$ with sensitivity of $74.1 \%$, and Specificity of $60 \%$ (AUC $=0.621,95 \% \mathrm{Cl}: 0.378-0.865, \mathrm{P}=0.318$ ).

Table 1

Demographic characteristics of patients

\begin{tabular}{|ll|}
\hline Variable & Mean \pm SD \\
\hline Age, yr & $54.7 \pm 12.2$ \\
\hline Weight, kg & $70.8 \pm 12.4$ \\
\hline Height, cm & $169.3 \pm 8.3$ \\
\hline BMI & $24.6 \pm 3.1$ \\
\hline ICU stay, day & $3.1 \pm 1.1$ \\
\hline MV duration, hr & $28 \pm 19.1$ \\
\hline surgery duration, hr & $5.8 \pm 1.9$ \\
\hline Weaning time, hr & $16.9 \pm 19.2$ \\
\hline BMl: body mass index, MV: mechanical ventilation, \\
\hline
\end{tabular}

weaning time: time of ventilator discontinuation from anesthesia. 
Table 2

Comparison of extubation failure based on quantitative/ qualitative CLT and gargle test results

\begin{tabular}{|llll|}
\hline \multirow{2}{*}{ Test } & \multicolumn{2}{l}{ Extubation failure } & \multirow{2}{*}{ P value } \\
\cline { 2 - 3 } & Yes & No & \\
\hline Quantitative CLT (\%) & $1(4.2)$ & $23(95.8)$ & 0.647 \\
$\leq 15$ & $5(5.3)$ & $89(94.7)$ & \\
$>15$ & & & \\
Qualitative CLT & $1(11.1)$ & $8(88.9)$ & 0.385 \\
non-appropriate & $5(4.6)$ & $104(95.4)$ & \\
appropriate & & & 0.032 \\
Gargle test & $2(33.3)$ & $4(66.7)$ & \\
negative & $4(3.7)$ & $103(96.3)$ & \\
positive & & & \\
\hline Fisher's Exact Test, CLT: cuff leak test & \\
\hline
\end{tabular}

Table 3

Comparison of sensitivity, specificity, PPV, NPV and accuracy between qualitative/quantitative CLT and gargle test

\begin{tabular}{|c|c|c|c|c|c|c|c|}
\hline \multirow[t]{2}{*}{ Test } & \multicolumn{2}{|c|}{ Extubation failure } & \multirow[t]{2}{*}{ Sensitivity } & \multirow[t]{2}{*}{ Specificity } & \multirow[t]{2}{*}{ PPV } & \multirow[t]{2}{*}{ NPV } & \multirow[t]{2}{*}{ Accuracy } \\
\hline & Yes & No & & & & & \\
\hline Quantitative CLT. \% & 1 & 23 & $16.6 \%$ & $79.4 \%$ & $4 \%$ & $94.6 \%$ & $76.27 \%$ \\
\hline$\leq 15$ & 5 & 89 & & & & & \\
\hline \multicolumn{8}{|l|}{$\otimes 15$} \\
\hline Qualitative CLT & 1 & 8 & $16.6 \%$ & $92.8 \%$ & $11.11 \%$ & $95.4 \%$ & $88.98 \%$ \\
\hline $\begin{array}{l}\text { non-appropriate } \\
\text { appropriate }\end{array}$ & 5 & 104 & & & & & \\
\hline Gargle & 2 & 4 & $33.3 \%$ & $96.3 \%$ & $33.3 \%$ & $96.3 \%$ & $92.92 \%$ \\
\hline $\begin{array}{l}\text { negative } \\
\text { positive }\end{array}$ & 4 & 103 & & & & & \\
\hline
\end{tabular}


Five patients out of 118 patients we investigated were excluded from this study, as the performance of the gargle test was not feasible due to their anatomical problems. One hundred thirteen patients were analyzed, and sensitivity, specificity, positive predictive value (PPV), NPV, and accuracy were calculated for them.

After extubation, incidents such as laryngeal edema, and reintubation were associated with extended mechanical ventilation and increased morbidity in ICU patients. (5) Up to 20\% of reintubation, 24 to 72 hours after extubation has been reported in the ICU setting. (18) Reintubation increases up to $47 \%$ in head and neck trauma, after maxillofacial surgery, and extensive neck injuries. On the one hand difficult intubation, on the other hand, airway edema, critical conditions, and inappropriate measures can lead to irreversible damage and death. $(19,20)$

Zhou et al. (5) reported that CLT can accurately identify the patients at risk of airway complications after extubation and decrease the rate of laryngeal edema after extubation. However, the rate of reintubation did not change. They also found that prolonged intubation could cause laryngeal edema after extubation.

Miller et al.(15) reported that with a cuff leak of $110 \mathrm{ml}$, the PPV for stridor after performing CLT was 80 $\%$, and the specificity of the test was $99 \%$. In Jaber et al. study,(21) CLT with a cuff leak of $12 \%$ (130 ml), showed a sensitivity of $85 \%$, specificity of $95 \%$, PPV and NPV were respectively $69 \%$ and $98 \%$. De Bast et al.(13) demonstrated that the best cut-off value for air leaks was $15.5 \%$. The sensitivity of this test was $78 \%$, specificity was $72.1 \%$, PPV was reported as $25 \%$, and NPV was $96.1 \%$. They concluded that due to the low PPV of this test, it may not be used to delay extubation.

The rate of post-extubation stridor, laryngeal edema, and efficacy of the leak test varies in different studies. Our study, unlike Miller et al. and Jaber et al.'s studies, showed low sensitivity and PPV. Like these studies, the specificity and NPV of our study were also high. Low PPV in our study was consistent with De Bast's study. Although PPV was higher in the gargle test, it was generally low in all three tests; PPV of quantitative CLT, qualitative CLT, and gargle test was 4\%, 11.11\%, 33.3\% respectively. In other words, when these tests indicate the presence of laryngeal edema, it does not necessarily mean that the extubation of the patient will be unsuccessful. Essentially, evaluation of laryngeal edema and the severity of it in the presence of endotracheal tube is difficult. Before the removal of the tube, tests have significant false results, and it is not possible to have a definitive verdict. Furthermore, it has been demonstrated that several measurements done by different individuals have just an average consistency in identifying the extent of the edema. (8) In our study, all of the parameters including, sensitivity, specificity, PPV, NPV, and accuracy were superior in the gargle test compared to CLTs (Table 3). According to high accuracy, NPV, and specificity (92.92\%, 96.3\%, 96.3\%, respectively), the gargle test was more effective in detecting airway edema. Considering its nature, groups of nerves and muscles which control larynx function are being assessed in the gargle test. This special characteristic cannot be determined in quantitative and qualitative tests. Further, the gargle test was significantly better in the evaluation of the likelihood of successful extubation $(p<0.032)$ (Table 2$)$. 
Prinianakis et al. (7) demonstrated that CLT was associated with lower efficacy in critically ill patients who have undergone mechanical ventilation for at least 48 hours after surgery. They attributed this difference to increased leak due to reduced compliance or increased airway resistance in surgical patients.

The use of CLTs in selective patients is a higher value compared to non-selective patients. (9) CLT, regardless of measuring absolute volume or expiratory volume percentage, is a weak predictor for the diagnosis of airway edema and extubation. It is not recommended that CLTs be used as a reliable indicator for postponing extubation, or the initiation of specific treatments (14) Thus, researchers are trying to propose other reliable measures to detect airway edema. All proposed tests have their specific limitations. Ding et al. (10) have used ultrasound to diagnose airway edema. They observed that aircolumn width and air-column width difference (ACWD) were lower in the stridor group compared to the non-stridor group. However, Mikaeili et al. (22) did not support this finding. They concluded that concerning stridor prediction, air-column width, and ACWD have low sensitivity and specificity compared to CLT. Eventually, both studies concluded that both CLT and laryngeal ultrasound have low sensitivity and PPV in the prediction of stridor. Thus, they should be used with precautions. (22)

\section{Conclusion}

The gargle test is simple, cheap, and does not require any equipment. Assessment of the nerves and muscles of the larynx and pharynx is a unique point in the gargle test. This feature of the gargle test can also provide greater reliability for safe extubation and reduce adverse outcomes in difficult extubations compared to other tests, such as CLTs.

\section{Abbreviations}

ICU

Intensive Care Unit

CLT

Cuff Leak Test

GT

Gargle Test

PPV

Positive Predictive Value

NPV

Negative Predictive Value

ACWD

Air-Column Width Difference

\section{Declarations}




\section{Ethics approval and consent to participate}

This study protocol was approved by the Tehran University of Medical Sciences Research Ethics Committee (Approval number: IR.TUMS.IKHC.REC.1396.2004 ). Written informed consent was obtained from all the participants.

\section{Consent for publication}

Not applicable.

\section{Availability of data and materials}

The datasets used and/or analyzed during the current study are available from the corresponding author on reasonable request.

\section{Competing interests}

None authors to be declared.

\section{Funding}

This study was supported by the Tehran University of Medical Sciences. Fisher \& Paykel Healthcare Co, Ltd. The funding source was not involved in the collection, analysis, or interpretation of data.

\section{Authors' contributions}

M.T.B. performed study design. L.A. interpreted the data. A.A. supervised the statistical analysis. M.T.B. wrote the draft, and all the authors critically reviewed the manuscript and approved the final version of the manuscript. All authors read and approved the final manuscript.

\section{Acknowledgements}

We would like to thank the ICUs and research center staff at Imam Khomeini Hospital Complex for their supports in this study.

\section{References}

1. Patel, A.B., Ani, C., and Feeney, C. Cuff leak test and laryngeal survey for predicting post-extubation stridor. Indian J Anaesth 2015, 59: 96-102.

2. Epstein S. Decision to extubate. Intensive care med 2002, 28(5):535-46.

3. Lee, C.H., Peng, M.J., and Wu, C.L. Dexamethasone to prevent postextubation airway obstruction in adults: a prospective, randomized, double-blind, placebo-controlled study. Crit Care 2007, 11: R7279. 
4. Chung, Y.H., Chao, T.Y., Chiu, C.T. et al. The cuff-leak test is a simple tool to verify severe laryngeal edema in patients undergoing long-term mechanical ventilation. Crit Care Med 2006, 34: 409-414.

5. Zhou, T., Zhang, H.P., Chen, W.W. et al. Cuff-leak test for predicting postextubation airway complications: a systematic review. J Evid Based Med 2011, 4: 242-254.

6. Sandhu RS, Pasquale MD, Miller K, Wasser TE. Measurement of endotracheal tube cuff leak to predict postextubation stridor and need for reintubation. J Am Coll Surg 2000, 190: 682-687.

7. Prinianakis G, Alexopoulou C, Mamidakis E, Kondili E, Georgopoulos D: Determinants of the cuff-leak test: a physiological study. Crit Care 2005, 9:R24-R31.

8. Cavallone LF, Vannucci A. Extubation of the difficult airway and extubation failure. Anesth Analg 2013, 116(2):368-83.

9. De Backer D. The cuff-leak test: what are we measuring? Crit Care 2004, 1:1-3.

10. Ding LW, Wang HC, Wu HD, Chang CJ, Yang PC. Laryngeal ultrasound: a useful method in predicting post-extubation stridor. A pilot study. Eur Respi J 2006, 27(2):384-9.

11. Popat M, Mitchell V, Dravid R, Patel A, Swampillai C, Higgs A, Difficult Airway Society Extubation Guidelines Group. Difficult Airway Society Guidelines for the management of tracheal extubation. Anaesthesia 2012, 67(3):318.

12. Newmark JL, Ahn YK, Adams MC, Bittner EA, Wilcox SR. Use of video laryngoscopy and camera phones to communicate progression of laryngeal edema in assessing for extubation: a case series. $J$ Intensive Care Med, 2013, 1:67-71.

13. De Bast Y, De Backer D, Moraine JJ, Lemaire M, Vandenborght C, Vincent JL. The cuff leak test to predict failure of tracheal extubation for laryngeal edema. Intensive Care Med, 2002, 28:1267-72.

14. Kriner, E.J., Shafazand, S., and Colice, G.L. The endotracheal tube cuff-leak test as a predictor for postextubation stridor. Respir Care 2005, 50: 1632-1638.

15. Miller, RL, Cole, RP. Association between reduced cuff leak volume and postextubation stridor. Chest 1996, 110:1035-1040.

16. Chung YH, Chao TY, Chiu CT, Lin MC. The cuff-leak test is a simple tool to verify severe laryngeal edema in patients undergoing long-term mechanical ventilation. Crit Care Med 2006, 34(2):409-14.

17. Mehta S. Tracheal tube cuff pressure. Anaesthesia. 1989 Dec;44(12):1001-2.

18. Karmarkar S, Varshney S. Tracheal extubation. Continuing Education in Anaesthesia Critical Care \& Pain 2008, 8(6):214-20.

19. Kulkarni AP, Agarwal V. Extubation failure in intensive care unit: predictors and management. Indian $\mathrm{J}$ Crit Care Med 2008, 12(1):1-9.

20. Schmutz A, Dieterich R, Kalbhenn J, Voss P, Loop T, Heinrich S. Protocol based evaluation for feasibility of extubation compared to clinical scoring systems after major oral cancer surgery safely reduces the need for tracheostomy: a retrospective cohort study. BMC Anesthesiol 2018, 18(1):43.

21. Jaber S, Chanques G, Matecki S, Ramonatxo M, Vergne C, Souche B, Perrigault PF, Eledjam JJ. Postextubation stridor in intensive care unit patients. Intensive Care Med 2003, 29(1):69-74. 
22. Mikaeili H, Yazdchi M, Tarzamni MK, Ansarin K, Ghasemzadeh M. Laryngeal ultrasonography versus cuff leak test in predicting postextubation stridor. J Cardiovasc Thorac Res 2014, 6(1):25.

\section{Figures}

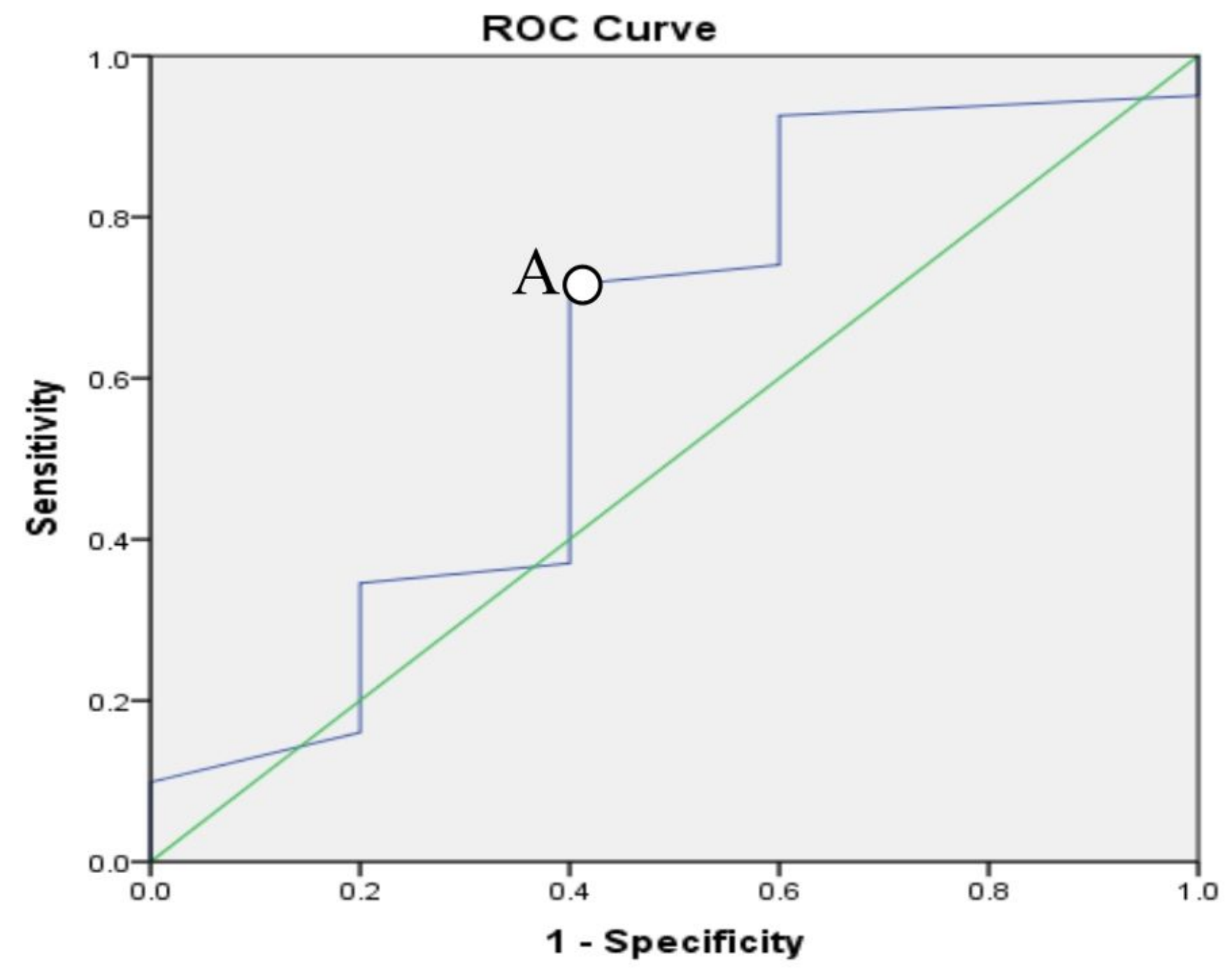

Diagonal segments are produced by ties.

Figure 1

Appropriate cut off point (A) of quantitative cuff leak test for extubation failure 